critical outlet diameter

$$
D_{m}=2 r_{m} \sin \theta
$$

where

$$
r_{m}=\frac{X_{b}-1}{X_{b}} \frac{Z_{0}}{\gamma Y_{b}}
$$

If the outlet diameter is less than $D_{m}$, the powder will form a stable dome across the outlet If the outlet is greater than or equal to $D_{m}$, the powder will flow through the outlet

\section{CONCLUSION}

The present theory makes it possible to determme approx1mately the stress distribution in an axially symmetric mass flow silo Furthermore the critical outlet diameter can be estımated by using eqn (34) Related to shear cell data acquired at Chr Michelsen Institute for several different powders [3], it was found that the critıcal outlet diameters arrived at by the present theory were usually about $20-30 \%$ less than when applying the standard Jenike [2] procedure This is consistent with what was found [1] for wedge-shaped hoppers

Acknowledgements - The author is indebted to the Royal Norwegian Councl for Industrial and Scientific Research for economic support and to Dr $R$ K Eckhoff for valuable advice in preparing the manuscript

Dept of Applied Physics

G ENSTAD

Chr Michelsen Institute

Nygaardsgt 114

N-5000 Bergen, Norway

\section{NOTATION}

$a, b, c, d$ given in eqns (27)-(30)

$D_{m}$ critical outlet diameter

$D$ diameter of the cylinder

$f_{c}$ unconfined fallure strength

$f_{c u}$ constant part of the falure function

$\Delta F$, supporting force from the wall exerted on a powder dome

$\Delta F_{2}$ net force exerted on a dome from the powder above and below

$h$ ratio between the mean stress below and above a discontinuity

$K$ ratio between the vertical and the horizontal stress in the powder close to the wall

$k$ coefficient in the linear fallure function

$R$ distance from vertex along the hopper wall to the transition

$r$ distance from vertex along the hopper wall

$r_{m}$ distance from vertex to the outlet

$\Delta r$ a tiny increment of $r$

$u$ given in eqn (25)

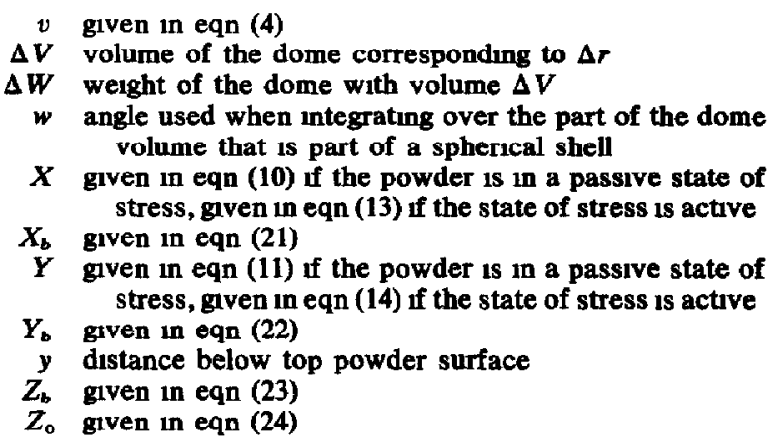

Greek symbols

$\alpha$ angle between the major principal stress at the wall and a line perpendicular to the wall when the powder is in a critical state of stress

$\alpha_{m}$ value of $\alpha$ giving maximum support regardless of the value of $\varphi^{\prime}$

$B$ angle between the direction of the major principal stress at the wall and a line perpendicular to the wall

$\beta_{a}$ active solution of eqn (3)

$\beta_{c} \quad \beta$ in the cylindrical part of the silo at the transition

$\beta_{h} \quad \beta$ in the hopper at the transition

$\beta_{p}$ passive solution of eqn (3)

$\gamma$ density of the powder

$\Delta$ discontinuous change of the durection of the major principal stress

$\delta$ effective angle of internal friction

$\theta$ inclination between the hopper wall and the vertical

$\sigma_{1}$ major principal stress

$\sigma_{2}$ minor principal stress

$\sigma$ mean stress

$\sigma=\frac{\sigma_{1}+\sigma_{2}}{2}$

$\sigma_{c}$ mean stress in the cylinder at the wall in the transition

$\sigma_{h}$ mean stress in the hopper at

the wall in the transition

$\sigma_{y}$ vertical stress at the wall in the cylinder

$\tilde{\sigma}_{1}$ major principal stress in a critical state

$\tilde{\sigma}_{2}$ minor principal stress in a critical state

$\varphi$ angle of internal friction

$\varphi^{\prime}$ angle of friction between powder and wall

where

the powder

is in a

fully

plastic

state

of

stress

REFERENCES

[1] Enstad G, Chem Engng Sct 1975301273

[2] Jenike A, Storage and Flow of Solids Bulletın 123, Utah University 1964

[3] Leversen P-G, Private communication. Chr Michelsen Institute

Chemucal Engineering Science 1977 Vol 32 pp $339-342$ Pergamon Press Printed in Great Britain

\title{
A note on capillary model developments for sandstone acidization
}

\author{
(Recetved 7 January 1976)
}

\section{INTRODUCTION}

A model has been developed and verified experimentally which predicts the movement of the acid and permeability fronts through linear porous sandstone cores [1-3] during matrix acidization This model has also been extended to describe the radial injection of $\mathrm{HF} / \mathrm{HCl}$ acid mixtures in sandstone reservors [4] In both the hinear and radial models, only three characteristic quantities (the Damkohler and acid capacity numbers and the porositypermeability relationship) need to be determined to completely describe the acidization process In this paper we shall show how pore size distribution may be utilized to evaluate the porositypermeability relationship and to estimate the surface area which is used in calculating the Damkohler number

Many models have been developed to describe the structure of porous media, the most common model is the capillary model in which the pore matrix is approximated by a bundle of straight, parallel capillaries of uniform radius running all the way through the porous core $[5,6]$ We will construct a pore size distribution using this type of model 


\section{EXPERIMIENTAL}

The acidization of the sandstone cores was carried out in a high pressure, high temperature permeameter, a full description of the apparatus and its operation has been reported[1] Mixtures of hydrofluonc and hydrochlonc acids were pumped at a constant rate through $1 \mathrm{un}$ dia cores The permeabulity of the core was measured as a function of time and samples of the effiuent acid were collected The strength and flow rate of the acid muxture, the length of the core, and the volume of acid injected were varied in the series of experiments After each experiment the core was retrieved, sectioned with a diamond saw, and parameters such as porosity, pore size distribution, mineral concentrations and permeability were measured for several sections The capullary pore size distributions of the acidized sandstone cores and the unacidized reference cores were measured using mercury injection The mercury injection was initiated at pressures well below atmospheric and therefore, large pores with radin up to $100 \mu \mathrm{m}$ could be measured Based on the surface tension and contact angle of mercury, the radius of a cylindrical pore which would be entered at an injection pressure can be calculated[5] from the equation

$$
r=1051 / P
$$

where the pressure and radius are given in psia and microns respectively

The cummulatıve void volume filled by the mercury is measured at increasing injection pressures The average of two mjection pressures allows a mean pore radius, $r_{m i}$ to be calculated and the change in the cummulative pore volume between the two injection pressures yields the corresponding pore volume, $V_{t}$ From the mean pore radius and the corresponding pore volume the pore size distribution can be calculated from a simple volume model for a bundle of cylinders

$$
V_{i}=n_{i} \overline{L_{i}} \pi\left(r_{m_{i}}\right)^{2}
$$

where $n_{i}=$ number of pores of radius $r_{m i}$ and $\bar{L}_{i}=$ mean pore length $n \bar{L}$

This can be rearranged to give the number length parameter,

$$
n \overline{L_{i}}=V_{i} / \pi\left(r_{m_{i}}\right)^{2}
$$

The number-length parameter can be calculated for each mean pore radus If the mean pore length is assumed to be constant regardless of the pore radus the number-length parameter approximates the relative number of pores at a given radus Similar use of the cylindrical pore volume model yields the pore surface area for each pore radius

\section{DISCUSSION OF RESULTS}

\section{(a) Macm and micro pores}

Figure 1 shows the pore size distribution number-length parameter for an acidized core and an unacidized core versus mean pore radius One observes that below three microns the curves are nearly identical while above three microns the number-length parameter is greater for the acidized cores This suggests that it is primarily, if not solely, the larger pores that are responsible for the increase in permeability resulting from acidization This increase (in relative pore number) can be accounted for in several ways-surface reaction at the pore wall increasing the radius of a pore, collision between two smaller pores forming one large pore, or the removal of debris from a construction in the pore

Macropores are referred to as those pores that are increased in number due to the acid, while micropores are pores that remain relatively constant in number during the acidization The threshold reactivity radius is the radius dividing the micro and macro pores of a given sandstone In order to obtain a better comparison of the pore size distribution for the acidized and unacidized we define the following parameter, which is the number-length ratio, $R$

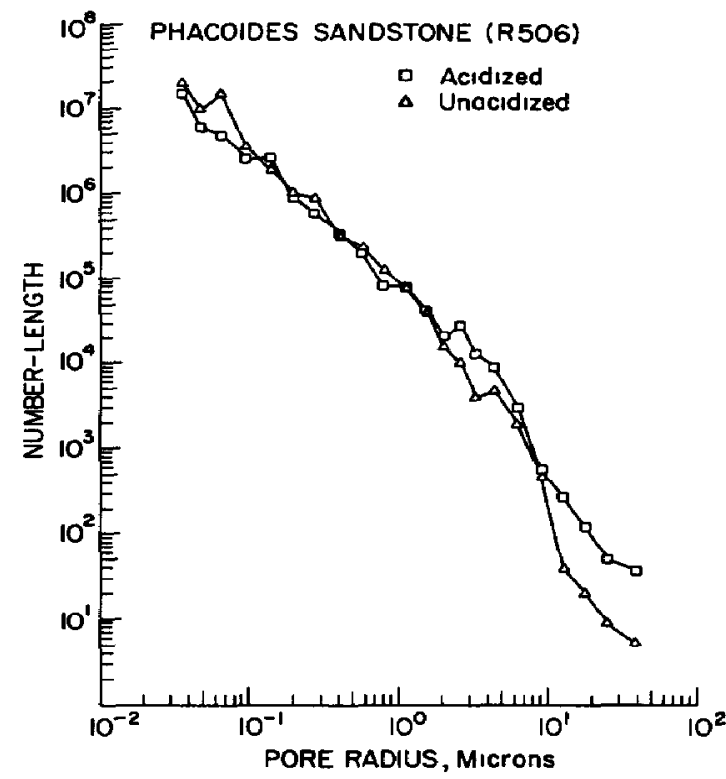

Fig 1 Relative pore number as a function of pore radius

$$
R=\frac{n \bar{L}(A)_{i}-n \bar{L}(U)_{i}}{n \bar{L}(U)_{i}}
$$

where $n \bar{L}(A)_{i}=$ number-length of pores of radius $r_{m i}$ after acidization and $n \bar{L}(U)_{i}=$ number-length of pores of radius $r_{m}$ before acidization If this ratio is zero, there has been no change in the relative pore number during the acidization process If the ratio is greater than zero an increase in the relative pore number has resulted and if it is less than zero there has been a decrease in relative pore number

Figures 2 and 3 presented in terms of number ratio as a function of sandstone pore radius show the greatest relative increase in pore number at radu greater than $26 \mu \mathrm{m}$ Below ths radius the ratio oscillates around zero, indicating little or no change in pore number This reactivity threshold radil between macro and micro pores for other acidization runs, which are shown in Table 1,

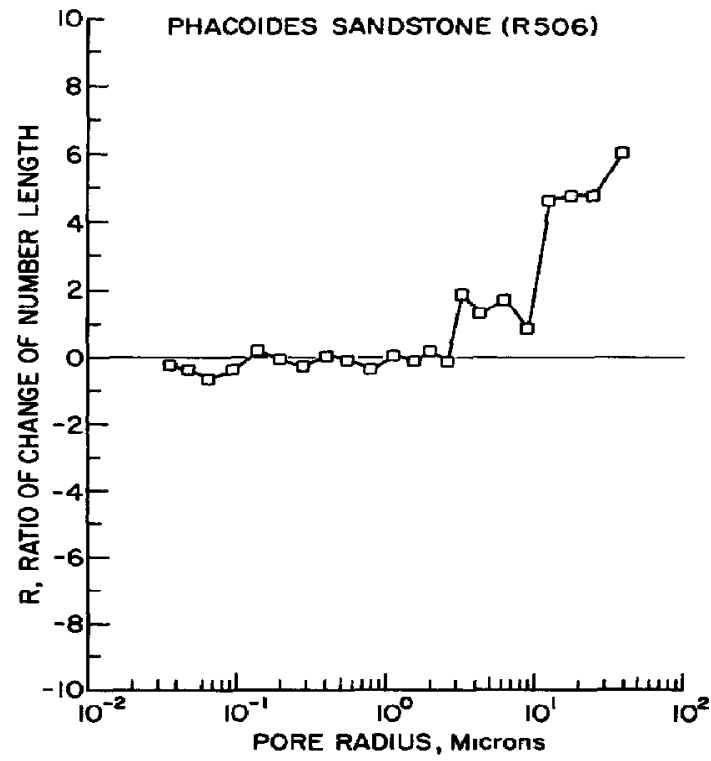

Fig 2 Relative change in pore number as a function of pore radius 


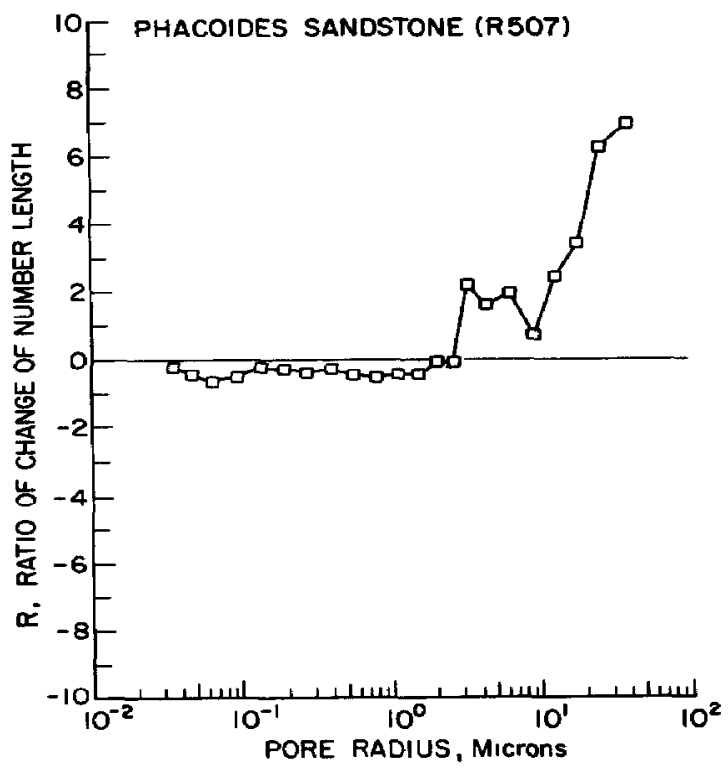

Fig 3 Relative change in pore number as a function of pore radius

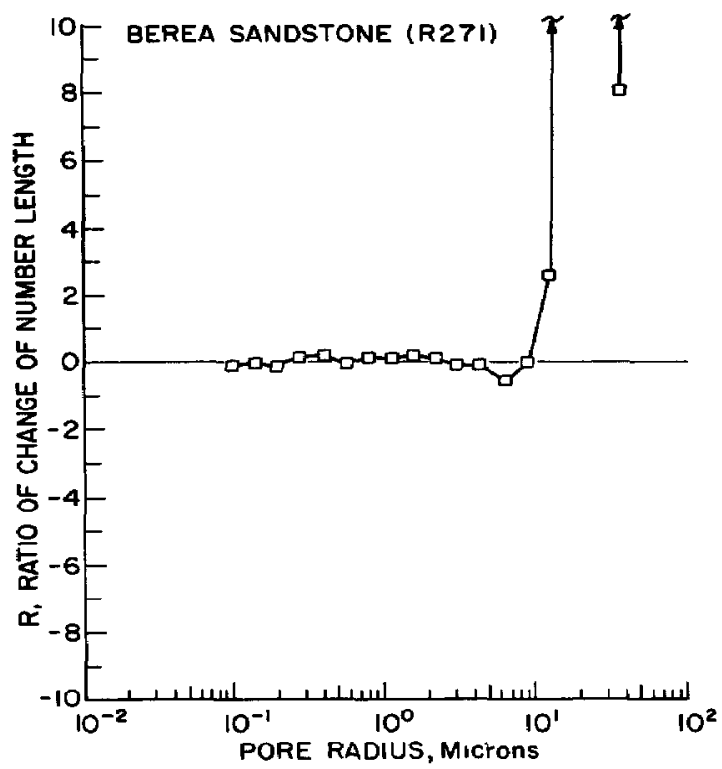

Fig 4 Relative change in pore number as a function of pore radius

Table 1 Macro and micro surface areas and threshold radius for acidized phacoides sandstone

\begin{tabular}{|c|c|c|c|c|c|}
\hline$\frac{\text { Run }}{\text { Number }}$ & $\begin{array}{l}\text { Threshold } \\
\text { Radius } \\
\text { (microns) }\end{array}$ & 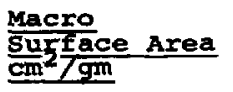 & $\begin{array}{l}\frac{\text { Micro }}{\text { surface Area }} \\
\frac{\mathrm{cm}^{2} / \mathrm{fm}}{\mathrm{gm}}\end{array}$ & $\begin{array}{l}\text { Macro* } \\
\text { surface } \\
\operatorname{cm}^{2} 7 g m\end{array}$ & e Area \\
\hline 436 & 43 & $4 B .7$ & 3620 . & 2438 & 8 \\
\hline $437 s-2$ & 3.3 & 529 & 3597 & 2410 & 0 \\
\hline $437 \mathrm{~s}-3$ & 43 & 363 & 3860 & 1862 & 2 \\
\hline 444 & 43 & 294 & 3920 & 2562 & 2 \\
\hline 466 & 43 & 326 & 3365 & 2262 & 2 \\
\hline 473 & 26 & 900 & 4394 & 2845 & 5 \\
\hline 502 & 26 & 745 & 2200 & 169 & \\
\hline 506 & 26 & 716 & 1700 & 2435 & 5 \\
\hline 507 & 26 & 77.1 & 1380 & 2029 & 9 \\
\hline 514 & 1.5 & 1179 & 2150 & 390 & 0 \\
\hline 515 & 28 & 800 & 1430 & 205 & \\
\hline average & 32 & & & & \\
\hline
\end{tabular}

* Based on threshola radius of 1 micron

undicates that for Phacordes sandstone the effectiveness of the acidization is greatest for those pores with a radu greater than $32 \pm 1 \mu \mathrm{m}$ Tests on other sandstones show this reactivity threshold radius to be unique for each sandstone type and must be experimentally determined in each case For example, the threshold radius for Berea Sandstone as shown in Fig 4 is $9 \mu \mathrm{m}$ The threshold radius can be used to relate changes between microscopic and macriscopic properties occurring durmg acidization

\section{(b) Surface area}

One of the three parameters in the model necessary to describe the movement of the acid in permeabilty fronts is the Damkohler number This number, which gives the shape of the acid and permeability profiles, is directly proportional to the reaction surface area Consequently it is of interest to determine the reaction surface area from the pore size distribution measurements and the threshold radius When accounting for changes is pore size distribution in terms of surface reaction phenomena, we should be concerned only with the effective surface area of the macropores rather than the total surface area As one observes from Table 1, the surface area for the macropores is substantually less than that for the micropores One can also use the experimentally determined values of the Damkohler number to estumate the reaction surface area When one considers the complexity of the acidization process along the relative simplicity of the model, the surface area calculated from the Damkohler number of $244 \mathrm{~cm}^{2} / \mathrm{gm}$ compares well with those values listed in Table 1 We should note that the areas shown in Table 1 for a threshold radius of $3 \mu \mathrm{m}$ will be somewhat lower than the actual area accessible to reaction since those pores with radi just below the threshold radius (say to $1 \mu \mathrm{m}$ ) will also provide some reactive surface area As one also notes from Table 1, the macro surface area based on a threshold radius of $1 \mu \mathrm{m}$ shows excellent agreement with the area obtained from experimental values of the Damkohler number[2] 


\section{(c) Porosty -permeabuluy relationshup}

Since changes in the pore size distribution are intrinsically related to changes in porosity, the capillary model should be able to calculate permeabulity changes $A$ relationship (based on steady state laminar flow through parallel pores) between pore size distribution and the porosity and the permeability has been developed by Schechter[7] The porosity and permeability are calculated from the first and second moments respectively of the pore size distribution Assuming that the geometric structure of the pore matrix is maintained during acidization, empirical geometric factors can be removed by taking the ratios of the property at any tume, $t$, to the initual value of the property The resulting relationships between pore size distribution and porosity is

$$
\frac{\phi(x, t)}{\phi_{\mathrm{o}}(x, 0)}=\frac{\iint L A W(A, L, x, t) \mathrm{d} A \mathrm{~d} L}{\iint \operatorname{LA} W(A, L, x, 0) \mathrm{d} A \mathrm{~d} L}
$$

The corresponding premeabilty relationship is

$$
\frac{K(x, t)}{K(x, 0)}=\frac{\iint L A^{2} W(A, L, x, t) \mathrm{d} A \mathrm{~d} L}{\iint L A^{2} W(A, L, x, 0) \mathrm{d} A \mathrm{~d} L}
$$

where $\phi(x, t)=$ porosity at position $x$ and tume $t, K(X, t)=$ permeability at position $x$ and time $t, L=$ pore length, $A=$ pore cross sectional area and $W(A, L, x, t)=$ pore density function

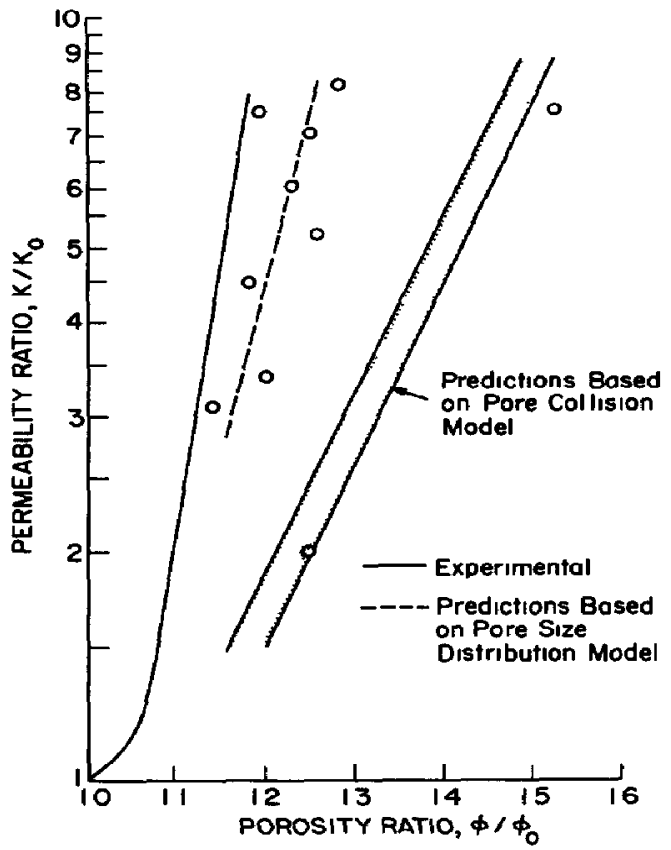

Fig 5 Local permeability as a function of porosity for Phacoides sandstone

†Present address Department of Chemical Engineering, Un1versity of Minnesota, Minneapolis, MN 55455, U S A

¥0 whom correspondence should be addressed
Application of the capllary model allows the product of the pore density function and the length to be replaced by number length, $n \bar{L}_{i}$, and the area by $\pi r_{m i}^{2}$. The resulting equation for finite changes of pore radus is

$$
\frac{K(x, t)}{K(x, 0)}=\frac{K}{K_{0}}=\frac{\sum r_{m i}^{4} n \bar{L}_{i}\left(r_{m p}, x, t\right)}{\sum r_{m i}^{4} n \bar{L}_{i}\left(r_{m p}, x, 0\right)}
$$

In calculating the permeability the sum is carned out only over the macro-pores as determined by the threshold radius

Knowledge of the porosity-permeability relationship is extremely critical in the design of matrix acid stimulation jobs [3, 4] We can now determine this relationship for Phacoides sandstone from the pore size distribution, the threshold radius, and the utulization of equs (5)-(7) Figure 5 compares the experumentally determined [8] local permeablity-porosity relationship with predictions based on the pore size distribution model, and the pore collision model[7] As one observes, the pore size distribution model agrees with the experimental results quite well

In summary, the hydrochloric and hydrofiuoric acid mixtures were found to have the greatest effect on pores in Phacoides sandstone with a radius greater than $3 \mathrm{\mu m}$ The division between the reactive macro-pores and the non reactive micro-pores, which is known as the threshold radus, was found to be unique for the two types of sandstones The pore size distribution model allows the prediction of the local permeability from the pore size distribution and the threshold radius Good agreement was found between the predicted porosity-permeahility relationship and the experimentally determined relationship The model and the threshold radius can also be used to determine the effectrve reaction surface area

\section{NOTATON}

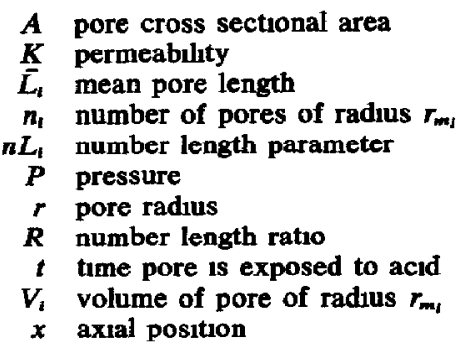

Greek symbol

$\phi$ porosity

Dept of Chemical Engineenng

University of Michrgan

Ann Arbor, MI 48109, US A

S T SWIFT $†$ H S FOGLERः

\section{REFERENCES}

[1] Lund $\mathbf{K}$, Fogler $H \mathrm{~S}$, McCune $\mathrm{C} C$ and Ault $J \mathbf{W}$, Chem Engng Scl 197631373

[2] Lund $K$ and Fogler H S, Chem Engng Scl 197631381

[3] Fogler H S and McCune C C, A I Ch EJ 197622799

[4] McCune C C, Cunningham J R, Fogler $H$ S, Lund $K$ and Ault J W, J Soc Petrol Engng 197515361

[5] Scheidegger A E, The Physics of Flow Through Porous Media MacMillan, New York 1957

[6] Burdine N T, Gournay L S and Reichertz P P, Petrol Trans AIME 1950189195

[7] Schechter R S and Gidley J L, A I Ch EJ 1969 15(3)

[8] McCune $C$ C and Fogler $H$ S, $A$ I Ch $E$ 74th National Meeting, Paper No 52c New Orleans, March 1973 